\title{
OBESIDADE EM FELINOS DOMÉSTICOS: FATORES DE RISCO, IMPACTOS CLÍNICOS, METABÓLICOS, DIAGNÓSTICO E TRATAMENTO
}

\author{
Camila Moura de Lima ${ }^{1}$ \\ Miriane Pereira Mendes ${ }^{2}$ \\ Anne Karoline da Silveira Flores ${ }^{2}$ \\ Gustavo Antônio Boff ${ }^{1}$ \\ Alexsander Ferraz ${ }^{1}$ \\ Mariana Cristina Hoeppner Rondelli ${ }^{3}$ \\ Márcia de Oliveira Nobre ${ }^{3}$
}

\begin{abstract}
RESUMO
O aumento da composição corporal pode promover alterações metabólicas e locomotoras, além de diminuir a expectativa de vida dos animais. Desse modo, o objetivo deste artigo é fornecer informações sobre a obesidade, elucidar os fatores de risco, diagnóstico, tratamento, assim como, ressaltar maneiras de enriquecimento ambiental e prevenção da obesidade. A obesidade possui origem multifatorial e pode contribuir para o surgimento de comorbidades. O diagnóstico baseia-se na avaliação nutricional e na identificação do grau de obesidade. Para isso existem algumas técnicas, como avaliação do peso, classificação do escore de condição corporal (ECC), índice de massa magra (IMM), raios-x de dupla energia (DEXA), tomografia computadorizada, ressonância magnética e métodos que auxiliam a estimar o índice de massa corporal dos felinos e o percentual de gordura. O tratamento consiste no planejamento nutricional, para isso deve-se realizada a troca de dieta para um alimento para perda de peso com densidade energética reduzida, baixo teor de gorduras e alto teor de proteínas e fibras. É fundamental que haja o retorno quinzenal ou mensal do paciente para a verificação da velocidade de perda e caso seja necessário realizar ajustes na dieta. Acrescido a dieta é essencial a introdução de enriquecimento ambiental para aumentar a atividade física. Diante do exposto, é de grande importância realizar o tratamento para obesidade, além de conscientizar os tutores sobre o correto manejo ambiental e nutricional, a fim de promover qualidade de vida a esses animais.
\end{abstract}

Palavras-chave: felinos, excesso de peso, má condição corporal, perda de peso, qualidade de vida.

\section{OBESITY IN DOMESTIC CATS: RISK FACTORS, CLINICAL, METABOLIC IMPACTS, DIAGNOSIS AND TREATMENT}

\begin{abstract}
The increase in body composition can promote metabolic and locomotor changes, in addition to decreasing the life expectancy of the animals. Thus, the objective of this article is to provide information about obesity, to elucidate risk factors, diagnosis, treatment, as well as to highlight ways of environmental enrichment and obesity prevention. Obesity has a multifactorial origin and can contribute to the emergence of comorbidities. The diagnosis is

\footnotetext{
${ }^{1}$ Doutoranda do Programa de Pós-Graduação em Veterinária da Universidade Federal de Pelotas. *Correspondência: camila.moura.lima@hotmail.com

${ }^{2}$ Graduanda em Medicina Veterinária Universidade Federal de Pelotas. mirimendes@hotmail.com

${ }^{3}$ Professora de clínica médica de pequenos animais na Universidade Federal de Pelotas. marianarondelli@gmail.com
}

Lima CM, Mendes MP, Flores AKS, Boff GA, Ferraz A. Rondelli MCH, Nobre MO. Obesidade em felinos domésticos: Fatores de risco, impactos clínicos, metabólicos, diagnóstico e tratamento. Vet. e Zootec. 2021; v28: 001-014 
based on nutritional assessment and identification of the degree of obesity. For this there are some techniques, such as weight assessment, classification of the body condition score (ECC), lean mass index (IMM), dual energy x-rays (DEXA), computed tomography, magnetic resonance and methods that help to estimate the feline body mass index and fat percentage. The treatment consists of nutritional planning. To do this, the diet must be changed to a weight loss food with reduced energy density, low fat content and high protein and fiber content. It is essential that there is a fortnightly or monthly return of the patient to check the speed of loss and if it is necessary to make adjustments to the diet. In addition to the diet, it is essential to introduce environmental enrichment to increase physical activity. Given the above, it is of great importance to carry out the treatment for obesity, in addition to making tutors aware of the correct environmental and nutritional management, in order to promote quality of life for these animals.

Key words: felines, overweight, poor body condition, weight loss, quality of life.

\section{OBESIDAD EN FELINOS DOMÉSTICOS: FACTORES DE RIESGO, IMPACTOS CLÍNICOS, METABÓLICOS, DIAGNÓSTICO Y TRATAMIENTO}

\section{RESUMEN}

El aumento de la composición corporal puede promover cambios metabólicos y locomotores, además de disminuir la esperanza de vida de los animales. Así, el propósito de este artículo es brindar información sobre la obesidad, dilucidar factores de riesgo, diagnóstico, tratamiento, así como resaltar formas de enriquecimiento ambiental y prevención de la obesidad. La obesidad tiene un origen multifactorial y puede contribuir a la aparición de comorbilidades. El diagnóstico se basa en la valoración nutricional y la identificación del grado de obesidad. Para ello existen algunas técnicas, como valoración de peso, clasificación de puntuación de condición corporal (ECC), índice de masa magra (IMM), rayos X de energía dual (DEXA), tomografía computarizada, resonancia magnética y métodos que ayudan a estimar el felino. índice de masa corporal y porcentaje de grasa. El tratamiento consiste en una planificación nutricional, para lo cual se debe cambiar la dieta por un alimento para adelgazar con densidad energética reducida, bajo contenido en grasas y alto contenido en proteínas y fibras. Es fundamental que exista un retorno quincenal o mensual del paciente para comprobar la velocidad de pérdida y si es necesario realizar ajustes en la dieta. Además de la dieta, es fundamental introducir un enriquecimiento ambiental para incrementar la actividad física. Por lo anterior, es de gran importancia realizar el tratamiento de la obesidad, además de concienciar a los tutores del correcto manejo ambiental y nutricional, con el fin de promover la calidad de vida de estos animales.

Palabras clave: felinos, sobrepeso, mala condición corporal, pérdida de peso, calidad de vida.

\section{INTRODUÇÃO}

A interação homem animal ocorre há muitos anos, sendo com os felinos domésticos (felis silvestris catus) a relação mais recente (1). Há relatos dessa relação desde o Egito, onde foram apreciados e sua imagem era bastante vista associada à religião da época, porém com o passar do tempo na idade média os felinos foram associados à bruxaria e nesse período houve uma baixa aceitação na sociedade. Logo, com o fim da idade média e o aparecimento de algumas doenças como a peste bubônica, os felinos foram bem vistos e utilizados para o controle de roedores (2). A partir disso, cada vez mais os felinos foram inseridos no cotidiano 
com os seres humanos e atualmente possuem um crescimento contínuo em sua população, sendo 24,7 milhões de felinos no Brasil (3). Esse crescimento pode ser explicado devido ao novo estilo de vida de algumas pessoas, pois esta espécie tende a se adaptar mais facilmente a ambientes pequenos quando comparada aos cães (3).

De acordo com a situação atual e com o desconhecimento por parte dos tutores de algumas características da espécie, é necessário fornecer aos tutores informações sobre as necessidades ambientais, nutricionais e comportamentais, a fim de evitar o aparecimento de alterações comportamentais e clínicas (4). Com base nisso, é de grande importância fornecer informações sobre as características da espécie, já que o comportamento alimentar difere-se dos cães (2). Os felinos domésticos descendem de seus ancestrais, que eram caçadores solitários, onde realizavam pequenas refeições diárias ao longo do dia e tinham um alto gasto calórico na busca de sua caça (5). Desse modo, os felinos domésticos são carnívoros estritos, e podem realizar de sete a 20 pequenas refeições se alimentados ad libitum (2). Com base nisso, é necessário estabelecer a frequência e a quantidade energética de acordo com as características corporais dos animais, a fim de prevenir o consumo excessivo de alimento e o aparecimento de algumas doenças nutricionais como, a obesidade $(6,7)$.

$\mathrm{O}$ excesso de peso é frequente na rotina clínica. A prevalência da obesidade felina pode variar de acordo com a localização geográfica (8). No Brasil há poucos estudos relatando a situação atual, porém em um estudo foi verificado que $14 \%$ dos felinos analisados estavam acima do peso (9). Já em outra pesquisa (10), analisou em um estudo de frequência da rotina cirúrgica, que cerca de $60,4 \%$ dos felinos estavam acima do peso. Logo, a obesidade é considerada a má condição clínica mais comum entre as alterações nutricionais em pequenos animais. Esse aumento na composição corporal pode promover alterações metabólicas e locomotoras, além de diminuir a expectativa de vida dos animais (8). Diante ao exposto, este artigo tem como objetivo fornecer informações sobre a obesidade, elucidar os fatores de risco, diagnóstico, tratamento, assim como, ressaltar maneiras de enriquecimento ambiental e prevenção da obesidade.

\section{DESENVOLVIMENTO}

\section{Definição de obesidade}

$\mathrm{O}$ aumento de peso acontece quando a ingestão de alimentos excede as necessidades energéticas diárias do animal. Com isso, há um balanço energético positivo crônico no organismo (11). O excesso de peso ocorre a partir do aumento de $10 \%$ a $15 \%$ do peso corporal ideal (12). Logo, a obesidade pode ser classificada em hipertrófica, onde os adipócitos aumentam de tamanho para armazenar maior quantidade de lipídeos. E hiperplásica, onde há o recrutamento de novas células e o aparecimento de novos adipócitos (13). A literatura relata que a obesidade hiperplásica geralmente ocorre quando há a superalimentação durante o período de crescimento dos animais, sendo esta considerada a mais difícil de tratar devido ao grande número de adipócitos (14).

\section{Tecido adiposo}

O tecido adiposo possui algumas funções, dentre elas, isolamento e proteção dos órgãos, manutenção do calor corporal, reservatório e fonte de energia e é considerado um órgão endócrino, que secreta hormônios e substâncias bioativas chamadas adipocinas (15). Sua composição caracteriza-se pela presença de adipócitos, fibras colágenas e reticulares, terminações nervosas, vasos sanguíneos, leucócitos, macrófagos, fibroblastos e pré-adipócitos (células indiferenciadas) (16). Ademais, pode ser dividido em tecido adiposo branco e tecido 
adiposo marrom, logo se diferem pela função e morfologia dos adipócitos, figura 01. Os adipócitos armazenam em seu interior triacilglicerol (TAG) $(16,17)$. Logo, o adipócito branco maduro carateriza-se por ser uma estrutura grande, porém seu tamanho pode variar conforme a quantidade de TAG em seu interior. Contudo, quando esses adipócitos não conseguem mais armazenar uma quantidade excessiva de TAG haverá um recrutamento de novas células chamadas pré-adipócitos e como consequência ocorrerá um aumento do tecido adiposo ocasionando a hiperplasia celular $(16,17)$.

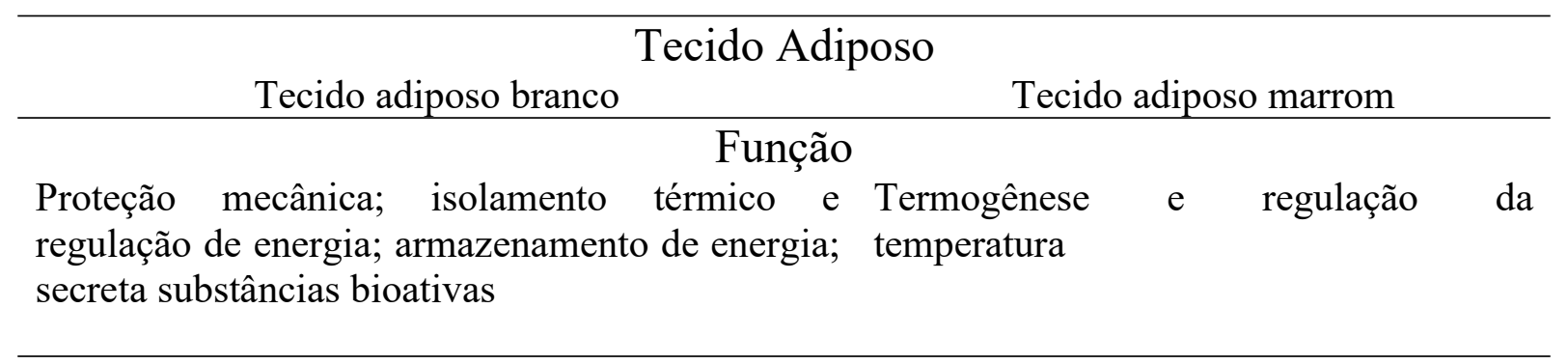

\section{Composição}

Presença de uma única gotícula lipídica e ocupa Presença de várias gotículas lipídicas grande parte da célula. com tamanhos variados

Figura 1. Especificações da diferença da função e composição do tecido adiposo branco e marrom.

\section{Adipocinas}

O tecido adiposo possui diversas funções no organismo do indivíduo, dentre elas, o papel de secretar substâncias chamadas de adipocinas. Logo com o aumento da gordura corporal há uma desregulação na concentração plasmática dessas substâncias promovendo assim malefícios ao organismo (15). Dessa forma, a obesidade pode proporcionar ao organismo humano e animal um distúrbio metabólico associado à inflamação crônica de baixo grau (18). A leptina é uma proteína pequena e é denominada uma adipocina pró-inflamatória, na qual expressa uma função importante na regulação do balanço energético estando diretamente relacionada com a regulação da homeostase da energia, manutenção da massa corpórea e em animais obesos sua concentração plasmática apresenta-se aumentada $(16,19)$. Desse modo, ela atua por intermédio da sinalização neuronal no núcleo arqueado hipotalâmico, onde ocorre a estimulação de neuropeptídeos que atuam inibindo a ingestão alimentar e aumentando o gasto energético e também atua em neuropeptídeos que aumentam a ingestão alimentar e reduzem o gasto energético $(20,21)$. Bem como, possui funções no sistema imune, angiogênese e fertilidade. Além disso, quando aumentada pode promover a desregulação dos osteoblastos no osso subcondral contribuindo para o acometimento das articulações (19).

A adiponectina é uma proteína sintetizada especificamente pelos adipócitos maduros e sua concentração plasmática está relacionada com o estado nutricional do paciente $(15,20)$. Logo, possui ação benéfica no organismo atuando como sensibilizador de transporte da glicose (Glut), potencializando assim a ação da insulina no organismo e contribuindo para o controle do metabolismo dos lipídios e carboidratos (19). Ademais, possui um papel importante anti-inflamatório, pois reduz a expressão do TNF- $\alpha$ e citocinas pró-inflamatórias, além de desempenhar uma ação benéfica nas articulações contribuindo para a proliferação de condrócitos e colágeno tipo II $(19,20)$. Porém em animais acima do peso sua concentração 
plasmática encontra-se diminuída, dessa forma, contribuindo para a diminuição do efeito benéfico no organismo (19).

O fator de necrose tumoral alfa (TNF- $\alpha$ ) é sintetizado pelos adipócitos, macrófagos, linfócitos e também por outras células (22). Logo, no tecido adiposo atua impedindo a lipogênese e também contribui para a lipólise. Com isso, animais acima do peso possuem um maior risco para o desenvolvimento de hiperlipidemias devido aos ácidos graxos circulantes (17). Além disso, interfere na sinalização de insulina, pois atua promovendo uma redução na expressão de (Glut4) (19). A interleucina-6 (IL-6) é uma citocina produzida por vários tipos de células como linfócitos $\mathrm{T}$ e $\mathrm{B}$, monócitos, macrófagos, adipócitos, dentre outras (23). Possui ação no sistema imune e ainda na resposta inflamatória, sendo uma das citocinas mediadoras da fase aguda da inflamação $(24,25)$. Bem como, no tecido adiposo é produzida pela gordura visceral e o aumento de IL-6 plasmática estimula a lipólise, desse modo, proporcionando a liberação de ácidos graxos e glicerol $(17,19)$.

\section{Fatores de risco}

O excesso de peso possui origem multifatorial, desse modo, é importante realizar a anamnese detalhada durante o atendimento clínico, a fim de identificar as possíveis causas, pois diversos fatores de risco podem estar envolvidos no aumento de peso (26). A literatura relata que dentre os fatores de risco para o desenvolvimento de obesidade apenas 3\% está relacionado com fatores diretamente do animal e $97 \%$ envolve hábitos, atitudes dos tutores e manejo alimentar (27).

Os fatores de risco específicos do animal podem envolver a predisposição genética, sendo que a raça british shorthair, manx, maine coon, persa e norwegian forest possuem maior predisposição de acordo com a literatura (28-30). Em relação à faixa etária, os animais de meia idade são mais propensos ao aumento de peso (29). No que se refere ao gênero, os felinos machos são mais propensos e possuem 1,3 mais chances em desenvolver sobrepeso quando comparado as fêmeas, e se forem castrados possuem 5,2 vezes mais chance, segundo alguns estudos $(29,31)$. Logo, a remoção dos hormônios sexuais circulantes pode influenciar no metabolismo, dessa forma, felinos castrados possuem 3,6 vezes mais chances em desenvolver obesidade $(17,31)$.

Já os fatores de risco associados com os hábitos, atitudes e manejo dos tutores com os animais compreendem o tipo de dieta, a quantidade fornecida e frequência de distribuição, características familiares, subestimação do escore de condição corporal e o desconhecimento por parte dos tutores sobre o comportamento felino podem predispor ao aumento de peso $(17,32)$. Logo, alimentos fornecidos à vontade, com alta palatabilidade, premium, rações terapêuticas, e de densidade energética elevada, se fornecidos de maneira errônea podem exceder as necessidades energéticas diárias dos animais e contribuir para o aumento de peso $(10,26,29)$.

As características familiares também podem influenciar no ganho de peso dos animais, como o tipo de moradia, ausência de crianças, outros animais e de acesso à rua. Desse modo, felinos que residem em apartamentos, ambientes pequenos e indoor estão em maior predisposição devido ao pequeno espaço para o gasto energético, dessa forma, tornando-os pouco ativos (30). A presença de crianças no ambiente domiciliar é considerada um fator redutor de risco, pois há uma maior interação com os animais propiciando o aumento da atividade física $(10,17)$. Bem como, a presença de outros animais pode auxiliar para o aumento da atividade física, porém casas com mais de quatro gatos podem contribuir para o aumento da ingestão de alimento devido à competição de alimento (33).

A subestimação do escore de condição corporal dos gatos por parte dos tutores pode proporcionar a superalimentação, pois muitos tutores possuem dificuldade em classificar o 
escore do seu animal de estimação (30). Em alguns estudos que compararam a percepção do escore atribuído pelo médico veterinário e pelo tutor demonstraram que a maioria dos tutores de gatos com sobrepeso e obesos tendem a subestimar o escore quando comparado ao atribuído pelo médico veterinário $(34,35)$. Ademais, o não entendimento por parte dos tutores sobre o comportamento felino pode contribuir para o ganho de peso, pois muitos comportamentos podem ser interpretados como pedido de alimento e com isso proporcionar a superalimentação e também a falta do conhecimento sobre as necessidades ambientais dos felinos pode contribuir para a promoção de um ambiente pouco atrativo e com isso diminuir o nível de atividade física $(4,17,36)$.

\section{Doenças associadas à obesidade}

O aumento de peso pode contribuir para o surgimento de comorbidades devido aos efeitos metabólicos, físicos e mecânicos que a obesidade pode proporcionar ao organismo $(8,17)$.

\section{Síndrome metabólica}

A síndrome metabólica (SM) em seres humanos é bem descrita e engloba uma série de fatores como diabetes mellitus, obesidade abdominal, aumento da pressão arterial e doenças cardiovasculares (37). Na espécie equina a SM é semelhante à humana e predispõe o desenvolvimento de laminite, hiperlipemia, osteocondrose e DM tipo 2 (38). No entanto, na espécie felina caracteriza-se por pelo menos duas destas alterações: hiperglicemia, hiperlipidemia, aumento da alanina aminotransferase (ALT) e redução da adiponectina (39).

Felinos com obesidade visceral possuem maior risco para desenvolver SM do que felinos com obesidade subcutânea (37). Esses mesmos autores relatam alguns critérios para diagnóstico de SM em felinos como, presença de doença associada a obesidade, acúmulo de gordura abdominal, pontuação de escore corporal superior ao ideal, aumento de glicose, triglicerídeos, colesterol e diminuição de adiponectina.

\section{Diabetes mellitus}

A diabetes mellitus (DM) é uma comorbidade associada com a obesidade, pois gatos acima do peso possuem mais de 3,9 vezes, chance de desenvolver DM quando comparados a felinos no escore ideal (40). Logo, em animais obesos o aumento de tecido adiposo contribui para o aumento da liberação de citocinas e diminuição de adiponectina fazendo com que haja uma interferência na sinalização de insulina, predispondo a resistência insulínica (41). Com base nesses efeitos os felinos desenvolvem DM tipo 2 (42). Ademais, casos de resistência insulínica podem ocasionar maior secreção de amilina, logo quando produzida em excesso a substância amilóide irá se depositar nas células fazendo com que haja uma disfunção das células beta $(\beta)$ pancreáticas e posteriormente a destruição das células $(43,44)$.

Vale ressaltar que a espécie felina possui a possibilidade de remissão diabética, isso ocorre quando o organismo possui a capacidade de manter a euglicemia sem a administração da insulina durante pelo menos quatro semanas em contrapartida sem aparecimento dos sinais clínicos (45). A remissão pode ocorrer de $50 \%$ a $70 \%$ em felinos diagnosticados precocemente e tratados corretamente, porém em felinos idosos e com doenças preexistentes as chances diminuem $(46,47)$. Para que isso ocorra estão envolvidos alguns fatores como, o diagnóstico precoce e controle glicêmico, utilização da correta insulina para a espécie, acompanhamento e monitoramento para o manejo de doses, introdução de uma dieta específica para controle glicêmico e redução do peso se o felino estiver com sobrepeso. Após 
a remissão deve ser realizado acompanhamento periódico do paciente para a avaliação clínica e realização de exames, com o intuito de minimizar a reincidência de DM $(48,49)$.

\section{Lipidose hepática}

A lipidose hepática (LH) é a enfermidade hepatobiliar mais comum em felinos domésticos. Caracteriza-se pelo acúmulo excessivo de triglicerídeos no fígado podendo assim comprometer a funcionalidade do órgão. Possui alta prevalência associada com os hábitos alimentares dos tutores e pets, sendo mais incidente em felinos obesos, dessa forma, felinos obesos possuem mais de 2,2 vezes chances de desenvolver lipidose quando comparados a felinos do escore ideal $(50,51) \mathrm{Na}$ obesidade os felinos estão mais propensos devido à ingestão excessiva de alimentos e como consequência maior produção de ácidos graxos e deposição nos hepatócitos. E também pode predispor a LH devido à ocorrência de lipólise em períodos de jejum (51).

\section{Doença do trato urinário}

O aumento do peso corporal pode predispor ao aparecimento de doenças do trato urinário dos felinos. Com isso, felinos obesos possuem mais de 1,5 vezes chances de desenvolver problemas urinários quando comparados ao escore ideal (51). Vale ressaltar que existem diversos fatores de risco para o aparecimento das doenças urinárias. Porém a relação com a obesidade pode estar associada pela dificuldade locomotora em decorrência do excesso de peso corporal e com isso a pouca utilização da caixa de areia (17). Dentre as principais doenças estão à doença do trato urinário inferior dos felinos, cistite idiopática, urolitíase, infecções no trato urinário e obstrução uretral $(52,53)$.

\section{Outras alterações}

O excesso de peso pode contribuir também para o aumento dos níveis de triglicerídeos e colesterol, bem como, alterações locomotoras, dermatopatias, doenças da cavidade oral e diminuição da resposta do sistema imune $(17,51)$.

\section{Diagnóstico}

O diagnóstico baseia-se na avaliação nutricional do paciente e na identificação do grau de obesidade (12). Para isso existem diversas técnicas, que podem contribuir para o diagnóstico, dentre elas, classificação do escore de condição corporal (ECC), índice de massa magra (IMM), avaliação do peso, raios-x de dupla energia (DEXA), tomografia computadorizada, ressonância magnética e também técnicas que auxiliam a estimar o índice de massa corporal dos felinos e o percentual de gordura (54-56). Diante disso, as técnicas de fácil aplicabilidade e baixo custo são bastante utilizadas na rotina clínica, pois os métodos que necessitam de anestesia acabam sendo pouco utilizados, pois ficam mais restritos para os locais onde há presença dos equipamentos e também devido ao alto custo $(11,17)$. Além disso, deve-se realizar exames laboratoriais para avaliar a saúde geral do paciente como hemograma, bioquímica sérica e exame de imagem quando necessário (11).

A classificação do escore de condição corporal (ECC) baseia-se na palpação das costelas, vértebras, asas do ílio, abdômen, cintura e na visualização da vista lateral e dorsal do paciente $(55,56)$. Apesar de ser uma avaliação subjetiva torna-se um método prático, de rápida aplicação e não invasivo, sendo assim bastante utilizado na rotina clínica (12). O percentual de gordura estimado pela avaliação do ECC na escala de 1 a 9 possui uma boa correlação com 
percentual de gordura encontrado pelo raio-x de dupla energia (DEXA) $(7,55)$. No ECC na escala de 1 a 9, a nota 5 é considerada o escore ideal e ECC $>5$ animais acima do peso (sobrepeso e obeso) (55,56). Logo, a cada ponto acima do escore ideal estima-se um acréscimo de 10 a 15\% do aumento de massa adiposa (55). Também é de grande importância realizar a avaliação do índice de massa muscular (IMM), onde avalia-se por intermédio da visualização e palpação dos músculos temporais, escapulares, vertebrais lombares, pélvicos e verifica-se se há ausência de perda muscular ou se há perda leve, moderada e acentuada $(21,56)$.

O peso corporal é um parâmetro que auxilia durante a avaliação nutricional e pode ser utilizado como uma ferramenta útil para o diagnóstico e acompanhamento de perda de peso $(12,56)$. É uma medida que estima a quantidade total das estruturas corpóreas, porém o peso ideal pode ser variável de acordo com a estrutura corporal e a raça do animal (Tabela 01) (12).

Tabela 1. Valor médio e desvio padrão de peso corporal de algumas raças de felinos domésticos.

\begin{tabular}{|c|c|c|}
\hline \multirow[t]{2}{*}{ Raças } & \multicolumn{2}{|c|}{ Peso médio ideal } \\
\hline & Valor mínimo & Valor máximo \\
\hline $\begin{array}{l}\text { Abissínio, American Shorthair, Angorá turco, } \\
\text { Bengal, British Longhair, Burmese Europeu, } \\
\text { Burmese Americano, Chartreux, Egyptian Mau, } \\
\text { Exótico de pelo curto, Maine coon, Norwegian } \\
\text { Forest, Oriental, Persa, Persa colourpoint (himalaio), } \\
\text { Ragdoll, Sagrado da Birmânia, Savannah, Siamês e } \\
\text { Sphynx }\end{array}$ & $3,7 \pm 0,8$ & $7,3 \pm 1,3$ \\
\hline
\end{tabular}

Fonte: Guia completo de raças felinas: tudo o que você precisa saber sobre os gatos (57).

O dexa caracteriza-se por utilizar raios-x de dupla energia, onde verifica-se a quantidade de mineral ósseo, massa muscular e adiposa (58). Desse modo, o princípio do método baseiase na emissão de raios- $\mathrm{X}$ com duas tensões $(70$ e $140 \mathrm{kVp})$ com duração entre cinco a dez minutos (20,58). É considerado um dos métodos de melhor exatidão e estimativa da composição corpórea in vivo de cães e gatos (20). Apesar de ser uma avaliação de melhor exatidão há algumas desvantagens para a aplicabilidade na rotina clínica, pois existe a necessidade da presença do equipamento e devido ao custo elevado é mais comum em centros de pesquisas, além disso, para a realização do exame o paciente necessita estar anestesiado (58). A tomografia computadorizada e ressonância magnética são métodos, em que é possível quantificar a gordura corpórea por intermédio da imagem, peça visualização dos locais de depósito de gordura. Porém são poucos utilizados na rotina clínica para diagnóstico de obesidade, pois há a necessidade de anestesia do paciente e também pelo alto custo (11).

Em seres humanos a aferição das medidas morfométricas é bastante utilizada para análise corporal, já em pequenos animais não há dados na literatura referenciando os valores ideais de circunferência torácica e abdominal devido à diferença na estrutura corporal entre as raças (58). Apesar disso, sabe-se que o percentual ideal de gordura corporal para gatos é $25 \%$ até $30 \%$, logo animais com mais de $30 \%$ são considerados acima do peso (12). Dessa forma, a aferição das medidas morfométricas pode auxiliar na estimativa da massa corporal, percentual de gordura do paciente e também durante o acompanhamento de perda de peso (54). Com isso, pode-se estimar o percentual de gordura corporal a partir do auxílio de uma fita métrica aferindo a circunferência torácica (nono espaço intercostal) e a distância entre a patela e a 
tuberosidade calcânea. Os valores dessas medidas podem ser dispostos na seguinte fórmula $\% \mathrm{GC}=(((\mathrm{CT} / 0,7067)-\mathrm{DCP}) / 0,9156)-\mathrm{DCP}$, onde o $\mathrm{CT}=$ circunferência torácica e o $\mathrm{DCP}=$ tamanho da patela até o calcâneo $(12,54)$. Já o índice de massa corporal felina pode-se obtido por intermédio da avaliação do peso corporal e da estatura em metros, onde mensurase o tamanho da coluna a partir da articulação atlanto-occipital até a última vértebra sacral, $(\mathrm{IMCF}=$ Peso $(\mathrm{kg}) /$ estatura(m) $)(59)$.

\section{Tratamento}

O tratamento consiste em um planejamento nutricional especifico para cada paciente, para isso deve-se realizar a troca de dieta para um alimento específico para perda de peso com densidade energética reduzida, baixo teor de gorduras e alto teor de proteínas e fibras, pois outros alimentos como o de manutenção e o ligth não suprem as necessidades nutricionais para uma perda de peso saudável (Tabela 02) (8). Com base no peso atual do paciente estimase a quantidade de perda de peso semanal de $0,5 \%$ a $1 \%$ (mínimo e máximo) (51). Logo, o protocolo de perda de peso inicia-se na estimativa do peso ideal do paciente, no qual pode ser estimado a partir da fórmula (peso ideal $=100 /(100+\% \mathrm{GC})$ x peso atual), após isso realiza-se a subtração do peso atual e estima-se a quantidade a ser perdida e também o tempo de tratamento (17).

Tabela 2. Comparação de alguns componentes nutricionais de alimento seco de manutenção, light e para perda de peso.

\begin{tabular}{lccc}
\hline Níveis de garantia & & Alimento industrializado & \\
& Manutenção & Light & Perda de peso \\
\hline Proteína bruta (\%) & $32 \%$ & $41 \%$ & $42 \%$ \\
Matéria fibrosa (\%) & $3,5 \%$ & $6,0 \%$ & $15 \%$ \\
Extrato etéreo (\%) & $17 \%$ & $9 \%$ & $10 \%$ \\
Quilocalorias (kcal) & $4,157 \mathrm{kcal}$ & $3,813 \mathrm{kcal}$ & $3,070 \mathrm{kcal}$ \\
\hline
\end{tabular}

*Valores nutricionais obtidos da ficha técnica no site comercial

A quantidade de alimento deve ser calculada de acordo com a necessidade energética de cada paciente por meio da fórmula $\left.(85 \times \text { (Peso atual })^{0,4} \mathrm{kcal}\right)$ (6). A introdução do novo alimento deve ser realizada de maneira gradual e a frequência de distribuição do alimento pode ser fornecida de acordo com a disponibilidade do tutor em fornecer o alimento, sendo recomendado dividir em pequenas porções ao longo do dia devido ao comportamento da espécie felina $(8,51)$. Ademais, pode ser realizada a introdução de alimento úmido para perda de peso e petiscos de baixa caloria, porém a quantidade não pode exceder $10 \%$ da necessidade energética do paciente. É fundamental que durante a perda de peso haja o retorno quinzenal ou mensal do paciente para a verificação da velocidade de perda e caso seja necessário realizar ajustes na dieta $(8,30,51)$.

Além da dieta é essencial a introdução de estratégias para aumentar a atividade física e o gasto calórico dos pacientes (36). Para isso, há uma diversidade de estratégias, que podem ser introduzidas na rotina do felino por meio de enriquecimento ambiental (EA) (36). O EA é um tema bastante discutido atualmente e consiste na introdução ou na modificação ambiental tornando o ambiente mais atrativo, podendo ser social, cognitivo, sensorial, alimentar e físico (36). Dessa forma, é de grande importância durante a anamnese identificar as principais falhas ambientais e com base nisso introduzir ideias de EA. Dentre as principais formas de EA, que auxiliariam na perda de peso estão às atividades que estimulam a mobilibade do felino, como brinquedos que simulam a caça com penas ou pelos (36). Bem como jogos interativos com 
alimento, onde há a necessidade de estímulo da cognição e olfato do animal até conseguir o alimento, desse modo, contribuindo para que o paciente se alimente de forma gradual (42).

Outro fator essencial durante a perda de peso é a conscientização dos tutores frente ao estado de condição corporal do paciente e também a fidelização ao tratamento, pois é necessária a pesagem do alimento diariamente, realizar as modificações ambientais sugeridas pelo médico veterinário e estimular o felino a uma rotina de exercícios diários $(8,19)$. Após o final do tratamento a quantidade de alimento deve ser calculada novamente e a critério do médico veterinário a escolha do novo alimento podendo ser introduzido um alimento light, de manutenção ou até mesmo manter o alimento para perda de peso. Para essa decisão é necessário continuar acompanhando o paciente para observar se manterá ou ganhara peso com o novo alimento $(30,51)$.

\section{Prevenção}

A prevenção para o ganho de peso baseia-se na identificação e redução dos fatores de risco, na conscientização dos tutores sobre o escore corporal ideal para os animais, na introdução de enriquecimento ambiental, a fim de promover um ambiente mais atrativo e assim estimular a atividade física e o gasto energético (8). Bem como, é fundamental, que os tutores busquem orientação nutricional com um médico veterinário, com o intuito de promover uma dieta balanceada especifica para a fase de vida do animal e também o fornecimento da correta quantidade de alimento para não haver um desequilíbrio energético (12). Desse modo, é de grande importância realizar visitas periódicas ao veterinário para controle do peso, vacinações e também para a avaliação geral da saúde do animal (17).

\section{CONSIDERAÇÕES FINAIS}

A obesidade é um distúrbio nutricional bastante frequente na rotina clínica. Essa condição corporal é oriunda de diversos fatores associados ao animal e aos hábitos e atitudes dos tutores. Esse estado nutricional pode predispor ao aparecimento de diversas comorbidades, como a síndrome metabólica, diabetes mellitus, lipidose hepática, doença do trato urinário, hiperlipidemias, dermatopatias, alterações locomotoras e doença da cavidade oral. Diante disso, o excesso de peso contribui para a redução da expectativa e qualidade de vida do animal.

O diagnóstico consiste na detecção do grau de obesidade, onde pode ser avaliado durante o exame clínico por intermédio de técnicas de fácil aplicabilidade clínica. A partir do diagnóstico o clínico estabelece o planejamento de perda de peso com dieta hipocalórica associado com a prática regular de exercícios físicos por meio do enriquecimento ambiental. Em vista disso, é de grande importância realizar o tratamento para obesidade, além de conscientizar os tutores sobre o correto manejo ambiental e nutricional desses animais. Após o término do tratamento recomenda-se avaliações periódicas do paciente por médico veterinário, a fim de realizar ajustes na dieta se necessário, e também para o acompanhamento do peso corpóreo do animal.

\section{AGRADECIMENTOS}

Agradecimento a Capes pela concessão da bolsa, ao CNPq (308152/2019-0), e também ao Hospital de Clínicas Veterinária da Universidade Federal de Pelotas.

\section{REFERÊNCIAS}

Lima CM, Mendes MP, Flores AKS, Boff GA, Ferraz A. Rondelli MCH, Nobre MO. Obesidade em felinos domésticos: Fatores de risco, impactos clínicos, metabólicos, diagnóstico e tratamento. Vet. e Zootec. 2021; v28: 001-014. 
1. Paz JEG, Machado G, Costa FVA. Fatores relacionados a problemas de comportamentos em gatos. Pesqui Vet Bras. 2017;37(11):1336-40.

2. Mentzel RE. Desenvolvimento filogenético do felino doméstico. In: Faraco CB, Soares GM. Fundamentos do comportamento canino e felino. São Paulo: Medvep; 2013.

3. Associação Brasileira da Indústria de Produtos para Animais de Estimação [Internet]. São Paulo: ABINPET; 2019 [citado 15 Maio 2021]. Disponível em: http://abinpet.org.br/mercado/

4. Ellis SLH, Rodan I, Carney HC, Heath S, Rochlitz I, Shearburn LD, et al. AAFP and ISFM feline environmental needs guidelines. J Feline Med Surg. 2013;15(3):219-30.

5. Hamper B, Bartges J, Kirk C, Witzel A, Murphy M, Raditic D. As necessidades nutricionais únicas do gato: um carnívoro estrito. In: Little SE. O gato: medicina interna. Rio de Janeiro: Roca; 2016.

6. National Research Council. Nutrient requirements of dogs and cats. Washington, DC: The National Academy Press; 2006.

7. Laflamme DP. Obesity in dogs and cats: what is wrong with being fat? J Anim Sci. 2012;90(5):1653-62.

8. Murphy M. Obesity treatment. Vet Clin North Am Small Anim Pract. 2016;46(5):883-98.

9. Mendes-júnior AF, Passos CB, Gáleas MAV, Secchin MC, Aptekmann KP. Prevalência e fatores de risco da obesidade felina em Alegre-ES, Brasil. Semina Cienc Agrar. 2013;34(4):1801-6.

10. Alves RS, Barbosa RCC, Gheren MW, Silva LE, Souza HJM. Frequência e fatores de risco da obesidade em uma população de gatos domésticos no Rio de Janeiro. Braz J Vet Med. 2017;39(1):22-45.

11. Okada Y, Ueno H, Mizorogi T, Ohara K, Kawasumi K, Arai T. Diagnostic criteria for obesity disease in cats. Front Vet Sci. 2019;6(284):1-5.

12. Fabretti AK, Gomes LA, Kemper DAG, Chaves RO, Kemper B, Pereira PM. Clinical determination of the nutritional status of companion animals. Semina Cienc Agrar. 2020;41(5):1813-30.

13. Guimarães ALN, Tudury EA. Etiologias, conseqüências e tratamentos de obesidades em cães e gatos-revisão. Vet Not. 2006;12(1):29-41.

14. Dodson MV, Hausman GJ, Guan L, Du M, Rasmussen TP, Poulos SP, et al. Lipid metabolism, adipocyte depot physiology and utilization of meat animals as experimental models for metabolic research. Int J Biol Sci. 2010;6(7):691-9.

15. Lacerda MS, Malheiros GC, Abreu AOW. Tecido adiposo, uma nova visão: as adipocinas e seu papel endócrino. Rev Cient Fac Med Campos. 2016;11(2):25-31. 
16. Fonseca-Alaniz MH, Takada J, Alonso-Vale MI, Lima FB. O tecido adiposo como centro regulador do metabolismo. Arq Bras Endocrinol Metab. 2006;50(2):216-29.

17. Chaves GV, Mendes MLR, Jacob FRC, Alves SN. A obesidade no gato doméstico revisão de literatura. Rev Clin Vet. 2018;23(134):32-46.

18. Zak A, Siwinska N, Elzinga S, Barker VD, Stefaniak T, Schanbacher BJ, et al. Effects of equine metabolic syndrome on inflamation and acute-phase markers in horses. Domest Anim Endocrinol. 2020;72:1-8.

19. Pöppl AG, Hummel J, Vicente G. Obesidade e alterações endócrinas. In: Hummel J, Vicente G, organizadores. Tratado de fisioterapia e fisiatria de pequenos animais. São Paulo: Paya; 2018.

20. German AJ, Holden SL, Morris PJ, Biourge V. Comparison of a bioimpedance monitor with dual-energy x-ray absorptiometry for noninvasive estimation of percentage body fat in dogs. Am J Vet Res. 2010;71(4):393-8.

21. Barbosa RCC, Botelho CFM, Alves RS, Souza HJM. Comparação do índice de massa corporal felino com a concentração plasmática de leptina e escore de condição corporal para o diagnóstico de obesidade em gatos domésticos. Acta Sci Vet. 2018;46(1):1-6.

22. Prado WL, Lofrano MC, Oyama LM, Dâmaso AR. Obesidade e adipocinas inflamatórias: implicações práticas para a prescrição do exercício. Rev Bras Med Esporte. 2009; 15(5):378-83.

23. Oliveira CMB, Sokata RK, Issy AM, Gerola LR, Salomão R. Citocinas e dor. Rev Bras Anestesiol. 2011;61(2):255-65.

24. Varellal PV, Forte WCN. Citocinas: revisão. Rev Bras Alergia Imunopatol. 2011;24(4):146-54.

25. Sippel C, Bastian RMAM, Giovanella J, Faccin C, Contini V, Del Bosco SM. Processos inflamatórios da obesidade. Rev Aten Saude. 2014;12(42):48-56.

26. O'connell EM, Williams M, Holden SL, Biourge V, German AJ. Factors associated with overweight cats successfully completing a diet-basead weight loss programme: an observacional study. BMC Vet Res. 2018;14(1):2-9.

27. Bland IM, Guthrie-jones A, Taylor RD, Hill J. Dog obesity: Veterinary practices' and owners' opinions on cause and management. Prev Vet Med. 2010;94(3-4):310-5.

28. Corbee RJ. Obesity in show cats. J Anim Physiol Anim Nutr. 2014;98(6):1075-80.

29. Tarkosova D, Story MM, Rand JS, Svoboda M. Feline obesity: prevalence, risk factors, pathogenesis, associated conditions and assessment: a review. Vet Med. 2016;61(6):295307.

30. Verbrugghe A. Epidemiology of small animal obesity. In: Cline MG, Murphy M. Obesity in the dog and cat. Boca Raton: CRC Press; 2019. 
31. Courcier EA, Mellor DJ, Pendlebury E, Evans C,Yam PS. An investigation into the epidemiology of feline obesity in Great Britain: Results of a cross-sectional study of 47 companion animal practises. Vet Rec. 2012;171(22):560-5.

32. Öhlund M, Egenvall A, Fall T, Hansson-hamlin H, Röcklinsberg H, Holst BS. Environmental risk factors for Diabetes Mellitus in cats. $J$ Vet Intern Med. 2017;31(1):29-35.

33. Russel K, Sabin R, Holt S, Bradley R, Harper EJ. Influence of feeding regimen on body condition in the cat. J Small Anim Pract. 2000;41(1):12-7.

34. Coucier EA, O'higgins R, Mellor DJ, Yam PS. Prevalence and risk factors for feline obesity in a first opinion practice in Glasgow, Scotland. J Feline Med Surg. 2010;12(10):746-53.

35. Peron L, Rahal SC, Castilho MS, Melchert A, Vassalo FG, Mesquita LR, et al. Owner's perception for detecting feline body condition based on questionnaire and scores. Top Companion Anim Med. 2016;31(3):122-4.

36. Sadek T, Hamper B, Horwitz D, Rodan I, Rowe E, Sundahl E. Feline feeding programs Addressing behavioral needs to improve feline health and wellbeing. J Feline Med Surg. 2018;20(11):1049-55.

37. Okada Y, Kobayashi M, Sawamura M, Arai T. Comparison of visceral fat accumulation and metabolome markers among cats of varying BCS and Novel classification of feline obesity and Metabolic Syndrome. Front Vet Sci. 2017;4(17):1-9.

38. Frank N. Equine metabolic syndrome. Vet Clin North Am Equine Pract. 2011;27(1):7392.

39. Mori N, Kawasumi K, Suzuki T, Yamamoto I, Kobayashi M, Arai T. Establishment of temporary criteria for metabolic syndrome (MS) Diagnosis and Assessment of the occurrence rate of MS in cats. J Anim Vet Adv. 2012;11(5):615-7.

40. Parker VJ, Orcutt E, Love L. Pathophysiology of obesity: comorbidities and anesthetic considerations. In: Cline MG, Murphy M. Obesity in the dog and cat. Florida: CRC Press; 2019.

41. Prentki M, Nolan CJ. Islet $\beta$-cell failure in type 2 diabetes. J Clin Invest. 2006;116(7):1802-10.

42. Clarke DL, Wrigglesworth D, Holmes K, Hackett R, Michel K. Using environmental and feeding enrichment to facilitate feline weigth loss. J Anim Physiol Anim Nutr (Berl). 2005;89(1-2):427.

43. Henson MS, O'brien TD. Feline models of type 2 diabetes mellitus. ILAR J. 2006;47(3):234-42. 
44. Henson MS, Hegstad-davies RL, Wang Q, Hardy RM, Armstrong PJ, Jordan K, et al. Evaluation of plasma islet amyloid polypeptide and serum glucose and insulin concentrations in nondiabetic cats classified by body condition score and in cats with naturally occurring diabetes mellitus. Am J Vet Res. 2011;72(8):1052-8.

45. Hoelmkjaer KM, Spodsberg E-MH, Bjornvad CR. Insulin detemir treatment in diabetic cats in a practice setting. J Feline Med Surg. 2015;17(2):144-51.

46. Bennett N, Greco DS, Peterson ME, Kirk C, Mathes M, Fettman MJ. Comparison of a low carbohydrate-low fiber diet and a moderate carbohydrate-high fiber diet in the management of feline diabetes mellitus. J Feline Med Surg. 2006;8(2):73-84.

47. Marshall RD, Rand JS, Morton JM. Treatment of newly diagnosed diabetic cats with glargine insulin improves glycaemic control and results in higher probability of remission than protamine zinc and lente insulins. J Feline Med Surg. 2009;11(8):683-91.

48. Lewitt M, Strage W, Church D. An dividual approach to feline diabetes care: a case report and literature review. Acta Vet Scand. 2016;58(1):49-54.

49. Behrend E, Holford A, Lathan P, Rucinsky R, Schulman R. 2018 AAHA diabetes management guidelines for dogs and cats. Vet Pract Guidelines. 2018;54(1):1-21.

50. Armstrong PJ, Blanchard G. Hepatic lipidosis in cats. Vet Clin North Am Small Anim Pract. 2009;39(3):599-616.

51. Vasconsellos RS, Borges NC, Carciofi AC. Obesidade em cães e gatos: elaboração do plano diagnóstico e terapêutico. In: Jericó MM, Kogika MM, Andrade JP. Tratado de medicina interna de cães e gatos. Rio de Janeiro: Roca; 2015.

52. Defauw PAM, Van de maele I, Duchateau L, Polis IE, Saunders JH, Daminet S. Risk factors and clinical presentation of cats with feline idiopathic cystitis. J Feline Med Surg. 2011;13(12):967-75.

53. Lund HS, Krontveit RI, Halvorsen I, Eggertsdóttir AV. Evaluation of urinalyses from untreated adult cats with lower urinary tract disease and healthy control cats: predictive abilities and clinical relevance. J Feline Med Surg. 2013;15(12):1086-97.

54. Butterwick R. How fat is that cat? J Feline Med Surg. 2000;2(2):91-4.

55. Laflamme DP. Understandig and managing obesity in dogs and cats. Vet Clin Small Anim Pract. 2006;36(6):1283-95.

56. WSAVA Nutritional Assessment Guidelines Task Force Members, Freeman L, Becvarova I, Cave N, MacKay C, Nguyen P, et al. WSAVA nutritional assessment guidelines. J Small Anim Pract. 2011;52(7):385-96.

57. Premierpet. Guia completo de raças felinas: tudo o que você precisa saber sobre gatos. São Paulo: PremieRpet ${ }^{\circledR} ; 2013$. 
58. Santarossa A, Parr JM, Verbrugghe A. The importance of assessing body composition of dogs and cats and methods available for use in clinical practice. J Am Vet Med Assoc. 2017;251(5):521-9.

59. Shields M, Tremblay MS, Gorber SC, Janssen I. Abdominal obesity and cardiovascular disease risk factors within body mass index categories. Health Rep. 2012;23(2):7-15.

Recebido em: 19/04/2021

Aceito em: 04/10/2021

Lima CM, Mendes MP, Flores AKS, Boff GA, Ferraz A. Rondelli MCH, Nobre MO. Obesidade em felinos domésticos: Fatores de risco, impactos clínicos, metabólicos, diagnóstico e tratamento. Vet. e Zootec. 2021; v28: 001-014 